\title{
Temporal Courses in EEG Theta and Alpha Activity in the Dynamic Health Qigong Techniques Wu Qin Xi and Liu Zi Jue
}

\author{
Diana Henz* and Wolfgang I. Schöllhorn \\ Institute of Sport Science, University of Mainz, Mainz, Germany
}

Health Qigong is a common technique of Traditional Chinese Medicine applied to strengthen mental and physical health. Several studies report increases in EEG theta and alpha activity after meditative Qigong techniques indicating a relaxed state of mind. To date, little is known on the effects of dynamic Health Qigong techniques that comprise bodily movements on brain activity. In the current study, we compared

OPEN ACCESS

Edited by:

Sergio Machado,

Salgado de Oliveira University, Brazi

Reviewed by:

Penny C. Werthner, University of Calgary, Canada

Jorge Aparecido Barros,

Universidade Católica Dom Bosco,

Brazil

Mardi A. Crane-Godreau, Department of Microbiology \& Immunology, Geisel School of Medicine at Dartmouth, United States

*Correspondence:

Diana Henz

henz@uni-mainz.de

Specialty section: This article was submitted to Movement Science and Sport

Psychology,

a section of the journal

Frontiers in Psychology

Received: 07 May 2017 Accepted: 18 December 2017 Published: 08 January 2018

Citation: Henz D and Schöllhorn WI (2018) Temporal Courses in EEG Theta and Alpha Activity in the Dynamic Health

Qigong Techniques Wu Qin Xi and Liu Zi Jue. Front. Psychol. 8:2291.

doi: 10.3389/fpsyg.2017.02291 effects of two dynamic Health Qigong techniques on EEG brain activity. Subjects performed the techniques Wu Qin Xi (five animals play) and Liu Zi Jue (six healing sounds) in a within-subjects design. Eyes-open and eyes-closed resting EEG was recorded before and immediately after each 15-min practice block. Additionally, the Profile of Mood States (POMS) questionnaire was administered at pretest, and after each 15-min practice block. Results show a decrease in alpha activity after $15 \mathrm{~min}$, followed by an increase after 30 min in the Health Qigong technique Liu Zi Jue. Theta activity was decreased after $15 \mathrm{~min}$, followed by an increase after $30 \mathrm{~min}$ in the technique Wu Qin $X i$. Results of the POMS indicated an increased vigor-activity level with decreased fatigue and tension-anxiety levels in both techniques after $30 \mathrm{~min}$ of practice. Our results demonstrate different temporal dynamics in EEG theta and alpha activity for the Health Qigong techniques Wu Qin Xi and Liu Zi Jue. We hypothesize that the found brain activation patterns result from different attentional focusing styles and breathing techniques performed during the investigated Health Qigong techniques.

Keywords: EEG, health Qigong, dynamic Qigong, theta activity, alpha activity

\section{INTRODUCTION}

Health Qigong is a technique of Traditional Chinese Medicine (TCM) applied commonly to strengthen physical and mental health. In several studies on meditative Qigong practice beneficial effects on health have been observed (for an overview see Ng and Tsang, 2009). Effects of Qigong practice on electrocardiographic parameters (Lee et al., 2000, 2003), blood pressure (Lee et al., 2003; Cheung et al., 2005), and breathing frequency (Sun, 1988) were investigated. Positive effects of Qigong practice on mental health were shown in anxiety disorders (Lee et al., 2004; Abbott and Lavretsky, 2013; Chan et al., 2013), posttraumatic stress disorders (Grodin et al., 2008; Kim et al., 2013), major depression (Tsang et al., 2003; Wang C. W. et al., 2013; Wang F. et al., 2013; Yeung et al., 2013; Yin and Dishman, 2014; Liu et al., 2015; Martínez et al., 2015), in the burnout syndrome (Stenlund et al., 2009, 2012), and in tinnitus 
(Biesinger et al., 2010). In recent studies, a stress alleviating effect was shown in healthy practitioners (Posadzki et al., 2010; Terjestam et al., 2010; Glei et al., 2012; Sousa et al., 2012; Hwang et al., 2013; Shim, 2014; Wang et al., 2014).

An essential research question is how the beneficial effects of Qigong practice on physical and mental health are mediated by neurophysiological processes. Several studies using electroencephalography (EEG) and fMRI demonstrated changes of brain activity induced by Qigong meditation. Most studies report increases in theta and alpha oscillations after Qigong meditation as a correlate for a relaxed and attentive mind. The first studies on the effect of Qigong meditation on electrical brain activity reported alpha activity predominantly in the anterior brain regions (Wallace, 1970). More differentiated results of effects of meditational Qigong techniques on EEG activity are shown dependent on expertise level. Several studies demonstrated shifts in alpha activation from posterior to anterior regions during Qigong meditation (Zhang et al., 1988a,b; Jang et al., 2004; Qin et al., 2009). Yang et al. (1994) report effects of Zhanzhuang Qigong on brain activity. After 1 year of practice, the alpha index of the right frontal and right temporal regions increased significantly. The beta activity of the right frontal and right temporal regions decreased significantly. A synchronization of brain activity was observed. The effects were not observed after half a year of continuing meditation. Thus, it was a gradually adjusting process.

Psychophysiological states of wakefulness and arousal as measured in terms of activation of particular EEG frequency bands are commonly related to distinct self-reported experiences in regular Qigong meditators. Mostly, an increase of alpha activity is related to an experience of relaxation and well-being, whereas an increase of EEG theta activity is correlated to a self-report of mindfulness. One of the main aims in Buddhist meditation traditions is to reach the state of mindfulness, which is defined as an attentive state of mind (for an overview see Tomasino et al., 2014).

For instance, Faber et al. (2012) demonstrated EEG alpha2 activity in posterior right parietal Brodmann areas 5, 7, 31, and 40 during Qigong meditation. It is argued that the found patterns of brain activation reflect self-reference, attention, and input-centered processing in Qigong meditation. Lee et al. (1997) investigated effects of ChunDoSunBup Qi-training on brain activity. The Qi-training comprises sound exercises, bodily motion, and meditation. Increases in alpha activity in ChunDoSunBup Qi-training were shown in the occipital regions in eyes-open conditions. The increase in occipital alpha activity was correlated to less self-reported state anxiety. One line of argumentation is that in ChunDoSunBup Qi-training activity of the occipital cortex is reduced and the thalamus is influenced. In a study by Pan et al. (1994) frontal mid-line theta rhythm during the concentrative Qigong state compared to the state of mind reached by non-concentrative Qigong engagement was demonstrated. Shim (2012) reported theta activity centering around the frontal lobe parts in Qigong masters and decreased alpha activity compared to beginners. The authors argue that Qigong experts maintained more deeply internalized and relaxed theta activity in the frontal lobe which reflects an attentive state of mind. Qigong masters show efficiency in keeping a relaxed and attentive mind around central midline during meditation (see also Tei et al., 2006a,b, 2009).

On a more structural level, Lehmann et al. (2012) showed reduced functional connectivity between cortical sources in Qigong meditation with reduced functional interdependence between brain regions. The authors argue that the reported subjective experience of non-involvement, detachment, and letting go, as well as of all-oneness and dissolution of ego borders during meditation is mirrored in the found pattern of brain activity.

Cheng et al. (2010) demonstrated an effect of Qigong meditation on prefrontal activation. Practitioners showed in comparison to non-practitioners of Qigong meditation a significant decrease in deoxyhemoglobin levels suggesting an increase in prefrontal activation during Qigong meditation.

Two fMRI studies report changes in brain function under the state of Qigong during pain exposure in Qigong masters (Chan et al., 2006; Yu et al., 2007). Functional activation in the SII-insula region and other brain areas was reported, whereas a functional suppression under the state of Qigong meditation was observed. It is argued that the found functional suppression in brain regions may be responsible for the reduced pain sensation in Qigong masters under the Qigong state.

In summary, systematical effects of static Qigong meditation on EEG brain activity are demonstrated with most studies reporting increases in frontal theta and posterior alpha activity as a correlate for a relaxed and attentive mind.

Qigong comprises different techniques that are commonly divided into static and dynamic forms. Static forms comprise meditational techniques whereas dynamic forms afford bodily movements as a tool to direct practitioners' attention to reach a meditative state (Tsang et al., 2002). To our knowledge, there are no systematical studies on effects on brain activity of dynamic Qigong techniques that afford bodily movement. In an experimental study conducted in our working group, increases in theta and alpha activity over the whole scalp after physical training of the Qigong technique Wu Qin Xi were observed (Henz and Schöllhorn, 2017). The same pattern of results was replicated in two consecutive studies on the effects on brain activity in the Qigong technique $W u$ Qin Xi (Henz et al., 2014, 2015). From a qualitative point of view, the found brain activation patterns were in line with findings of studies conducted with static meditational Qigong. As in studies on static meditational Qigong, increased fronto-central theta, and posterior alpha activity was observed.

The main aim of the present study is to investigate acute effects on EEG brain activity in the dynamic Health Qigong techniques Liu Zi Jue (six healing sounds) and Wu Qin Xi (five animals play) in a comparative experimental design. The Health Qigong technique Liu Zi Jue is the art of expiration in producing six different sounds [xu (嘘), he (呵), hu (呼), si (呬), chui (吹), xi (嘻)]. Each sound is performed within a specific movement routine without moving the feet, with each sound and movement routine repeated six times. Inverse abdominal respiration is required while performing the practice, as well as producing the six sounds respectively during exhaling. While inhaling with lips closed and tongue reaching the palate, one 
should breath naturally through the nose and make the abdomen uplifted (Chinese Health Qigong Association, 2007). According to TCM theory moving lips and teeth with different forces can affect different organs and the circulation of Qi and blood in the vessels and in the meridians, this results in training of the organs, harmonizing the Qi and blood, and balancing the Yin and Yang (Jiang and Zou, 2013). Its diaphragmatic breathing may produce increased asynchronous and paradoxical breathing movements, and its prolonged expiration and slowing of the breathing rate is widely used and produces a satisfactory effect (for an overview see Yang and $\mathrm{Wu}, 2011)$. The Health Qigong technique $W u$ Qin Xi comprises a consecutive sequence of complex movement configurations. These configurations are the game of the five animals (tiger, deer, bear, monkey, bird) with each movement sequence performed between two and three times for several minutes. Practitioners are requested to focus on breathing when performing the movement sequences. According to theoretical assumptions of TCM $W u$ Qin Xi is an intervention to strengthen especially physical health in general (for an overview see Yang and $\mathrm{Wu}, 2011$ ).

Only a few studies have been conducted to investigate the effects of Liu Zi Jue and Wu Qin Xi on physical and mental health. Studies on the dynamic Qigong technique Liu Zi Jue report beneficial effects on chronic obstructive pulmonary disease (Xiao and Zhuang, 2015), on pain consciousness, and on depression (Dong and Lee, 2013). Positive effects of Wu Qin Xi training are reported on lumbar spinal disease (Yeom et al., 2013; You et al., 2013, 2014; Zhang et al., 2014), blood lipid levels and the antioxidant enzyme activities (Chen, 2011).

To date, there are no systematical studies reported on the effects of Liu $\mathrm{Zi}$ Jue Qigong training on EEG brain activity that examine the underlying neuronal processes that mediate the positive effects on physical health. Recently, it was shown that Liu Zi Jue training made beneficial effects on pain consciousness and depression of elderly single women (Dong and Lee, 2013). As depression is correlated with reduced EEG alpha activity (see Başar et al., 2012), one hypothetical assumption could be derived that Liu Zi Jue might have a modulating effect on EEG brain activity which is one neurophysiological substrate for a subjective experience of increased well-being.

A second aim of the present study is to investigate the temporal course of EEG brain activity in both Qigong techniques. To our knowledge there are no systematical studies on the temporal course of EEG brain activity in the dynamic Qigong techniques $W u$ Qin Xi and Liu Zi Jue. One important question considering the design of interventions to achieve an optimum effect is the acute duration of Qigong practice. Does the theta and alpha activity increase with increasing practice duration? A further important research question is whether differences in the temporal course of EEG brain activity occur in the dynamic Qigong techniques $W u$ Qin $X i$ and Liu Zi Jue. In previous studies on Qigong meditation modulations of brain activity were observed already after a short-term practice of 10 (e.g., Faber et al., 2012) to $15 \mathrm{~min}$ (Lavallee et al., 2011; Shim, 2012).

From previous studies on Qigong meditation, we suppose that practicing the Qigong techniques Wu Qin Xi and Liu Zi Jue result in increased EEG theta and alpha activity. According to the theoretical framework of TCM, we hypothesize that practicing the Qigong technique Liu $\mathrm{Zi}$ Jue leads to stronger increases in EEG theta activity as a correlate for a concentrative meditational state compared to practice of the Qigong technique $W u$ Qin Xi. From a physiological perspective, we argue that in Liu Zi Jue the role of breathing is more reinforced due to producing the six sounds when performing the movements. Therefore, breathing behavior tends to be more regulated by adapting to the sound production than in $W u$ Qin Xi. Recent EEG studies have shown that abdominal breathing techniques lead to increased frontal theta activity (e.g., Yu et al., 2011; Chervin et al., 2012; Park and Park, 2012). Considering breathing as a meditation technique, it was observed that Shaolin Dan Tian Breathing increases EEG frontal theta activity (Chan et al., 2011). The authors argue that the observed increase in frontal theta activity in Shaolin Dan Tian Breathing is a correlate for an attentive mind.

Another line of argumentation for a stronger increase in EEG theta activity in Liu $\mathrm{Zi}$ Jue than in Wu Qin Xi is that attentional processes are more guided by directing the focus of attention on breathing due to sound production than on mere movement performance which might lead to stronger internalized attention reflecting in increased frontal theta activity. Van der Lubbe et al. (2014) showed that the focus of spatial attention leads to changes in EEG brain activity. Additionally, movement configurations in $\mathrm{Liu} \mathrm{Zi}$ Jue are reported from practitioners to be less complex than in $W u$ Qin Xi. Therefore, practitioners possibly tend to spend more effort on control of breathing than on control of complex movement configurations as in Wu Qin Xi.

From this line of argumentation and on basis of previous studies on the effect of abdominal breathing techniques, we expect a stronger increase in frontal EEG theta activity in Liu $\mathrm{Zi}$ Jue than in $W u$ Qin Xi. From results of previous studies on Qigong meditation and Wu Qin Xi (Henz et al., 2014, 2015; Henz and Schöllhorn, 2017) we hypothesize effects in Liu Zi Jue and $W u$ Qin Xi after 15 min with increases in theta activity in Liu Zi Jue after 30 min compared to Wu Qin Xi.

The aims of the present study are:

1. The analysis of the spontaneous eyes-closed and eyes-open EEG spontaneous activity before and after training of the dynamic Qigong techniques Wu Qin Xi and Liu Zi Jue.

2. Comparison of the temporal course of EEG brain activity after a 15-, and a 30-min practice of the Qigong techniques Wu Qin $X i$ and Liu Zi Jue.

\section{METHODS}

\section{Participants}

Twenty subjects (mean age 32.8 years, age range $21-50,8$ males, 12 females) volunteered in this study. Subjects were recruited from the Qigong workshops at the Institute of Sports Science of the University of Mainz and from sports science courses and had at least regular Qigong training experience of 1 year. The subjects were all healthy, and had no current diseases or a history of neurological impairments or intake of medication that may have affected EEG recordings. All subjects were naïve as to the purpose of the current study. All subjects gave written informed 
consent. The experimental procedures were approved by the local ethics committee at the Johannes Gutenberg University of Mainz, Germany. All experimental procedures were carried out in accordance with the Declaration of Helsinki.

\section{Experimental Procedure}

The subjects were sat comfortably in a dimly-lit isolated room. At each measurement time point, participants began with a resting condition. Spontaneous EEG of the subject was recorded for $2 \mathrm{~min}$ for eyes-open, and $2 \mathrm{~min}$ for eyes-closed conditions. Then, subjects were required to perform two consecutive 15min Qigong practice sessions. The experiment contained two tasks: Participants were required to perform the dynamic Qigong technique Liu $\mathrm{Zi}$ Jue and $W u$ Qin $X i$ in a within-subjects design at 2 consecutive days. The Qigong technique Liu Zi Jue consisted of six distinct movement routines, with each movement routine repeated six times. In the Qigong technique $W u$ Qin $X i$, participants performed the five movement sequences tiger, deer, bear, monkey, and bird. Participants were required to coordinate their breathing with the prescribed movements in both Qigong conditions. Each of the both Qigong techniques was performed on a separate day. Experimental conditions were randomized. All training sessions, were performed with eyes-open. EEG data were obtained during the six resting conditions: (1) pretraining ( $W u$ Qin Xi) rest, (2) pretraining (Liu Zi Jue) rest, (3) post-Qigong ( Wu Qin Xi) practice after 15 min, (4) post-Qigong (Wu Qin Xi) practice after $30 \mathrm{~min}$, (5) post-Qigong ( Liu Zi Jue) practice after $15 \mathrm{~min}$, (6), post-Qigong (Liu Zi Jue) practice after $30 \mathrm{~min}$, which were then used for subsequent analyses.

To assess the psychological mood, the Profile of Mood States (POMS) questionnaire (McNair et al., 1971) was submitted to the subjects before the experiment, and after each experimental task. The assessment of mood was focused on vigor-activity, fatigue-inertia, and tension-anxiety.

\section{EEG Recording Details}

Electroencephalography (EEG) was recorded by using the Micromed Brainquick amplifier and Micromed Brainspy software (Micromed, Venice, Italy). Recordings were made from Fp1, Fp2, F3, F7, Fz, F4, F8, C3, Cz, C4, T3, T4, P3, P7, Pz, P4, $\mathrm{P} 8, \mathrm{O} 1, \mathrm{O} 2$ placed according to the international 10-20 system with reference to the nose. All electrode impedances were kept at $10 \mathrm{k} \Omega$ or below. The EEG signals were continuously recorded and digitized at a sampling rate of $256 \mathrm{~Hz}$. The EEG signal was amplified with a fixed time constant of $0.3 \mathrm{~s}$ with a high-pass filter at $0.5 \mathrm{~Hz}$, and a low-pass filter at $120 \mathrm{~Hz}$ (frequency range: $0.5-120 \mathrm{~Hz}$ ). Electrooculography (EOG) was monitored placed at the medial upper and lateral orbital rim of the right eye (time constant: $0.3 \mathrm{~s}$; high pass filter: $0.1 \mathrm{~Hz}$; low pass filter: $120 \mathrm{~Hz}$; frequency range: $0.5-120 \mathrm{~Hz}$ ).

\section{EEG Analysis \\ Spontaneous EEG Analysis}

The spontaneous EEG was recorded before and after the Qigong interventions for $2 \mathrm{~min}$ with eyes-closed, and $2 \mathrm{~min}$ eyes-open conditions. Subsequent analyses were performed separately for eyes-closed and eyes-open conditions. The EEG and EOG signals were visually evaluated and portions of the data that contained aberrant eye movements, muscle movements of artifacts were removed. The EEG was analyzed and Discrete Fast Fourier Transform was used to obtain the mean power amplitudes in theta $(4-7.5 \mathrm{~Hz})$, low-frequency alpha- $(8-10 \mathrm{~Hz})$, highfrequency alpha-2 $(10-12.5 \mathrm{~Hz})$, beta $(13-29.5 \mathrm{~Hz})$, and gamma $(30-40 \mathrm{~Hz})$ bands. The ranges of high- and low-frequency alpha bands were defined according to previous studies by Aeschbach et al. (1999), and Cantero et al. (2002).

\section{Statistical Analysis}

A statistical comparison of power of theta, alpha-1, alpha2, beta, and gamma bands was done by repeated-measure analyses of variance (ANOVA) including the within-subject factors as Qigong technique (Wu Qin Xi, Liu Zi Jue), time (pretest, post-Qigong $15 \mathrm{~min}$, post-Qigong $30 \mathrm{~min}$ ), experimental condition (eyes-open, eyes-closed), and location (Frontal, Central, Temporal, Parietal, Occipital). Additionally, partial eta-squared $\left(\eta_{p}{ }^{2}\right)$ was calculated to determine effect sizes. ANOVAs were followed by Bonferroni corrected post-hoc tests for further comparisons. POMS data were subjected to repeatedmeasure ANOVAs including the within-subject factors as Qigong technique ( $W u$ Qin Xi, Liu Zi Jue), and time (pretest, post-Qigong $15 \mathrm{~min}$, post-Qigong $30 \mathrm{~min}$ ). Additionally, partial eta-squared $\left(\eta_{p}^{2}\right)$ was calculated to determine effect sizes. Subsequently, Bonferroni corrected post-hoc tests were calculated for further comparisons. Effects were considered to be statistically significant when the $p$-values were less than 0.05 .

\section{RESULTS}

\section{Statistical Description: Spontaneous EEG}

Figures 1A,B show the mean power spectra for the theta, alpha1, alpha-2, beta, and gamma band in Liu Zi Jue and Wu Qin $X i$. Main statistical outcomes for the EEG data are presented in Table 1. The ANOVA of theta responses revealed highly significant differences for Qigong technique, $F_{(1,19)}=9.20$, $p=0.007, \eta_{p}{ }^{2}=0.33$. Post-hoc comparisons showed that the spontaneous EEG theta power was significantly higher in $\mathrm{Liu} \mathrm{Zi}$ Jue than in $W u$ Qin $X i, p=0.009$. The ANOVA of theta responses revealed significant differences for time, $F_{(2,38)}=4.16, p=0.023$, $\eta_{p}{ }^{2}=0.18$. Post-hoc comparisons showed that the spontaneous EEG theta power was significantly higher after $30 \mathrm{~min}$, than after $15 \mathrm{~min}, p=0.028$, and compared to the resting baseline, $p=0.015$. The ANOVA of theta responses revealed significant differences between locations, $F_{(4,76)}=3.304, p=0.015$, $\eta_{p}{ }^{2}=0.14$. Post-hoc comparisons showed that spontaneous EEG theta power at frontal, and central electrodes was higher than that of temporal, $p<0.05$ each, parietal, $p<0.05$ each, and occipital electrodes, $p<0.05$ each. Subsequent analyses showed that theta power was most increased in the frontal region at the electrode $\mathrm{Fz}, p<0.05$, and in the central region at electrode $\mathrm{Cz}, p<0.05$. The ANOVA of theta responses revealed significant results for technique $\times$ time, $F_{(2,38)}=4.72, p=0.020, \eta_{p}^{2}=0.18$. Posthoc comparisons showed that in Wu Qin Xi theta power was significantly higher after $30 \mathrm{~min}$ than in Liu Zi Jue, $p=0.01$. The ANOVA of theta responses revealed significant effects for 


\section{A Theta Power}
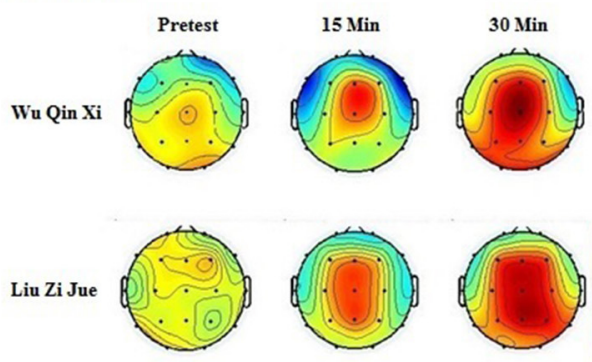

Alpha-1 Power
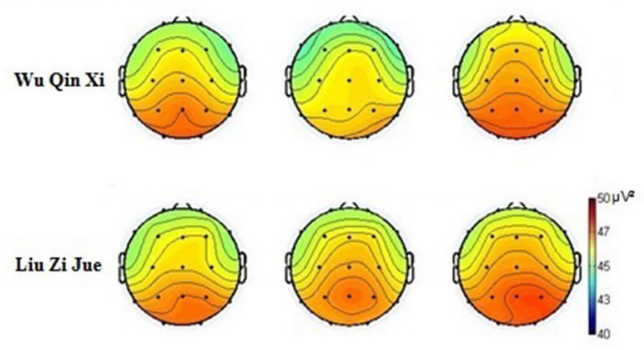

Alpha-2 Power
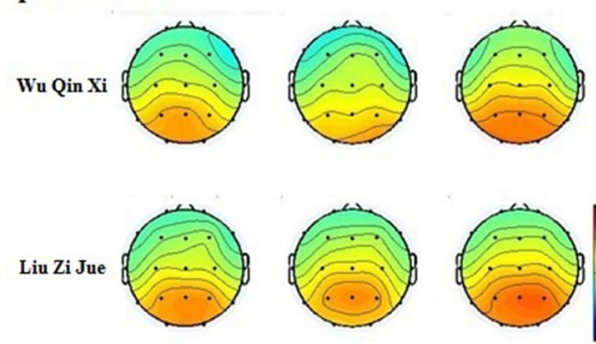

\section{B Beta Power}
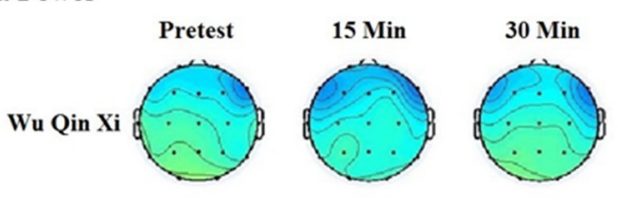

Liu Zi Jue
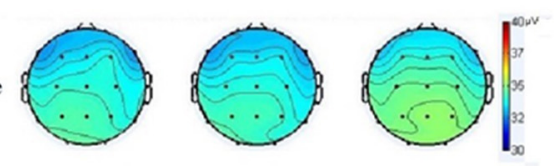

Gamma Power
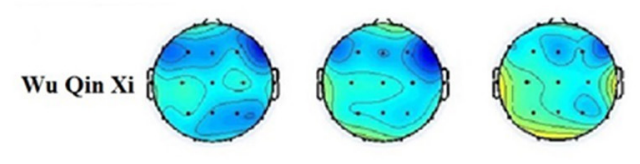

Liu Zi Jue
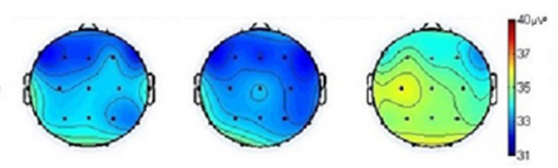

FIGURE 1 | (A) Spontaneous EEG theta, alpha-1, and alpha-2 activity at resting baseline, and after 15 and 30 min Qigong practice. Results show different temporal courses in EEG theta, alpha-1, and alpha-2 activity in the dynamic Health Qigong techniques Wu Qin Xi and Liu Zi Jue during practice. (B) Spontaneous EEG beta and gamma activity at resting baseline, and after 15 and 30 min Qigong practice. Results show different temporal courses in EEG gamma activity in the dynamic Health Qigong techniques Wu Qin Xi and Liu Zi Jue during practice.

technique $\mathrm{x}$ location, $F_{(4,76)}=3.029, p=0.023, \eta_{p}{ }^{2}=0.14$. Post-hoc comparisons showed increased theta power in $\mathrm{Liu} \mathrm{Zi}$ Jue in parietal locations, compared to $W u$ Qin $X i, p=0.016$. The ANOVA of theta responses revealed significant effects for time $\times$ location, $F_{(8,152)}=2.39, p=0.027, \eta_{p}^{2}=0.11$.

The ANOVA of alpha-1 responses revealed significant differences for Qigong technique, $F_{(1,19)}=7.45, p=0.013$, $\eta_{p}{ }^{2}=0.28$. Post-hoc comparisons showed that the spontaneous EEG alpha-1 power was higher in Liu Zi Jue, than in Wu Qin Xi, $p=0.017$. The ANOVA of alpha- 1 responses revealed significant differences for time, $F_{(2,38)}=4.86, p=0.011, \eta_{p}{ }^{2}=0.29$. Post-hoc comparisons showed that the spontaneous EEG alpha1 power was significantly higher after $30 \mathrm{~min}$, than after $15 \mathrm{~min}$, $p=0.021$, and compared to the resting baseline, $p=0.011$. There was no difference between resting baseline, and $15 \mathrm{~min}$. The ANOVA of alpha-1 responses revealed significant results for Qigong technique $\mathrm{x}$ time, $F_{(2,38)}=3.612, p=0.037$, $\eta_{p}{ }^{2}=0.16$. Post-hoc comparisons showed that in Wu Qin Xi alpha-1 power was significantly decreased after $15 \mathrm{~min}$ compared to $30 \mathrm{~min}, p=0.045$, and baseline rest, $p=0.014$. In Liu Zi Jue, alpha-1 activity was increased after $15 \mathrm{~min}$ compared to baseline, $p=0.027$, and after $30 \mathrm{~min}, p=0.034$. The ANOVA of alpha1 responses revealed significant differences between locations, $F_{(4,76)}=2.958, p=0.025, \eta_{p}{ }^{2}=0.14$. Post-hoc comparisons showed that spontaneous EEG alpha-1 power was higher at central, parietal, and occipital electrodes than that of frontal, and temporal electrodes, $p<0.05$ each. Subsequent analyses showed that alpha-1 power was most increased in the frontal region at the electrodes Fz, $p<0.05$, and F4, $p<0.05$.

The ANOVA of alpha-2 responses revealed significant differences for Qigong technique, $F_{(1,19)}=6.85, p=0.017$, $\eta_{p}{ }^{2}=0.20$. Post-hoc comparisons showed that the spontaneous EEG alpha-2 power was significantly higher in Liu Zi Jue, than in $W u$ Qin $X i, p=0.05$. The ANOVA of alpha-2 responses revealed significant differences for time, $F_{(2,38)}=4.66, p=0.016$, $\eta_{p}{ }^{2}=0.23$. Post-hoc comparisons showed that the spontaneous EEG alpha-2 power was significantly higher after $30 \mathrm{~min}$, than after $15 \mathrm{~min}, p=0.025$, and compared to the resting baseline, 
TABLE 1|P-values and partial eta-squared $\left(\eta_{p}{ }^{2}\right)$ for the EEG data for the factors Qigong technique, time, location, and the technique $\times$ time, technique $\times$ location, and time $\times$ location interactions, Statistical values are depicted for the theta, alpha-1, alpha-2, beta, and gamma band.

\begin{tabular}{|c|c|c|c|c|c|c|c|c|c|c|c|c|}
\hline & \multicolumn{2}{|c|}{ Qigong technique } & \multicolumn{2}{|c|}{ Time } & \multicolumn{2}{|c|}{ Location } & \multicolumn{2}{|c|}{ Technique $\times$ Time } & \multicolumn{2}{|c|}{ Technique $\times$ Location } & \multicolumn{2}{|c|}{ Time $\times$ Technique } \\
\hline & $p$ & $\eta_{p}^{2}$ & $p$ & $\eta_{p}^{2}$ & $p$ & $\eta_{p}^{2}$ & $p$ & $\eta_{p}^{2}$ & $p$ & $\eta_{p}^{2}$ & $p$ & $\eta_{p}^{2}$ \\
\hline Theta & 0.007 & 0.33 & 0.023 & 0.18 & 0.015 & 0.25 & 0.020 & 0.18 & 0.023 & 0.14 & 0.027 & 0.11 \\
\hline Alpha-1 & 0.013 & 0.28 & 0.011 & 0.29 & 0.025 & 0.14 & 0.037 & 0.16 & n.s. & - & n.s. & - \\
\hline Alpha-2 & 0.017 & 0.20 & 0.016 & 0.23 & 0.032 & 0.11 & 0.028 & 0.13 & n.s. & - & n.s. & - \\
\hline Beta & n.s. & - & n.s. & - & n.s. & - & n.s. & - & n.s. & - & n.s. & - \\
\hline Gamma & 0.032 & 0.11 & 0.018 & 0.19 & 0.045 & 0.08 & 0.013 & 0.28 & n.s. & - & n.s. & - \\
\hline
\end{tabular}

$p=0.01$. The ANOVA of alpha- 2 responses revealed significant results for technique $\mathrm{x}$ time, $F_{(2,38)}=3.92, p=0.028, \eta_{p}^{2}=0.13$. Post-hoc comparisons showed that in Wu Qin Xi EEG alpha-2 power was decreased after $15 \mathrm{~min}$ compared to $30 \mathrm{~min}, p=0.035$, and after resting baseline, $p=0.018$. In Liu Zi Jue alpha-2 power was increased after $30 \mathrm{~min}$ compared to $15 \mathrm{~min}, p=0.041$, and resting baseline, $p=0.031$. There was no significant difference between $15 \mathrm{~min}$, and resting baseline. The ANOVA of alpha2 responses revealed significant differences between locations, $F_{(4,76)}=2.799, p=0.032, \eta_{p}{ }^{2}=0.11$. Post-hoc comparisons showed that spontaneous EEG alpha-2 power was higher at parietal and occipital electrodes, than that of frontal, central, and temporal electrodes, $p<0.05$ each. Subsequent analyses showed that alpha-2 power was most increased in the parietal region at the electrode $\mathrm{P} 4, p<0.05$, and in the occipital region at electrode $\mathrm{O} 2, p<0.05$.

The ANOVA of beta responses revealed no significant differences for Qigong technique, time, experimental condition, neither for locations.

The ANOVA of gamma responses revealed significant differences for Qigong technique, $F_{(1,19)}=5.33, p=0.032$, $\eta_{p}{ }^{2}=0.11$. The ANOVA of gamma responses revealed significant differences for time, $F_{(2,38)}=4.45, p=0.018, \eta_{p}^{2}=0.19$. Post-hoc comparisons showed that the spontaneous EEG gamma power was significantly higher after $30 \mathrm{~min}$, than after $15 \mathrm{~min}$, $p=0.038$, and compared to the resting baseline, $p=0.011$. The ANOVA of gamma responses revealed significant results for technique $\mathrm{x}$ time, $F_{(2,38)}=4.92, p=0.013, \eta_{p}{ }^{2}=0.28$. Posthoc comparisons showed increased gamma power in Liu Zi Jue after $30 \mathrm{~min}$ compared to $W u$ Qin $X i$ at each measurement point, $p=0.019$. Further, in Liu Zi Jue gamma power was significantly higher after $30 \mathrm{~min}$, than after $15 \mathrm{~min}, p=0.021$, and compared to baseline rest, $p=0.009$. There was no difference in gamma activity between baseline rest and after $15 \mathrm{~min}$ in Liu Zi Jue. The ANOVA of gamma responses revealed significant differences between locations, $F_{(4,76)}=2.56, p=0.045, \eta_{p}^{2}=0.08$. Posthoc comparisons showed that spontaneous EEG gamma power at central electrodes was higher than that of frontal, parietal, temporal, and occipital electrodes, $p<0.05$ each.

\section{POMS Questionnaire}

Means and standard deviations of the POMS scales vigoractivity, fatigue-inertia, and tension-anxiety are depicted in Figure 2. Main statistical outcomes for the POMS scales are presented in Table 2. The ANOVA for the scale vigor-activity revealed significant differences for the factor Qigong technique, $F_{(1,19)}=5.46, p=0.022, \eta_{p}^{2}=0.18$, with a higher vigoractivity level in $W u$ Qin Xi than in Liu $Z i$ Jue, $p=0.02$. The ANOVA for the factor time showed significant differences for training duration, $F_{(2,38)}=3.97, p=0.034, \eta_{p}{ }^{2}=0.10$. Post-hoc comparisons revealed that the vigor-activity level was significantly higher after $30 \mathrm{~min}$ of practice than at pretest, $p=0.02$, and after $15 \mathrm{~min}$ of practice, $p=0.04$. The technique $\times$ time interaction was significant, $F_{(2,38)}=3.61, p=0.042$, $\eta_{p}^{2}=0.07$.

The ANOVA for the scale fatigue-inertia revealed no significant difference for the factor Qigong technique. The ANOVA for the factor time showed significant differences for training duration, $F_{(2,38)}=4.58, p=0.028, \eta_{p}^{2}=0.14$. Post-hoc comparisons showed that the fatigue-inertia level was significantly reduced after $15 \mathrm{~min}, p=0.02$, and $30 \mathrm{~min}$ of practice, $p=0.02$, compared to pretest. The technique $\mathrm{x}$ time interaction was significant, $F_{(2,38)}=3.82, p=0.040, \eta_{p}{ }^{2}=0.08$.

The ANOVA for the scale tension-anxiety revealed no significant effect for Qigong technique. A further ANOVA showed significant differences for training duration, $F_{(2,38)}=4.20, p=0.021, \eta_{p}^{2}=0.12$. Post-hoc comparisons showed that the tension-anxiety level was decreased after $30 \mathrm{~min}$ of practice compared to pretest, $p=0.02$, and 15 -min practice, $p=0.04$. The technique $\times$ time interaction was not significant.

\section{DISCUSSION}

The current literature includes several previous investigations on effects of static Qigong meditation on EEG brain activity. Most studies report an increase in EEG theta and alpha activity during and after static Qigong meditation. In the present study, we investigated effects of the dynamic Qigong techniques Wu Qin $X i$ and Liu Zi Jue with emphasis on the temporal course of brain activation at training durations of 15 and $30 \mathrm{~min}$, respectively. We demonstrated an increase of midline fronto-central theta and posterior alpha-1, and alpha-2 activity after practice of the dynamic Qigong techniques Wu Qin Xi and Liu Zi Jue. Our results mirror the findings of previous studies of effects on EEG brain activity after Qigong meditation. From a qualitative point of view, a comparable effect for the dynamic Qigong techniques $W u$ Qin Xi and Liu Zi Jue on EEG brain activity as found in 
A

Vigor-Activity

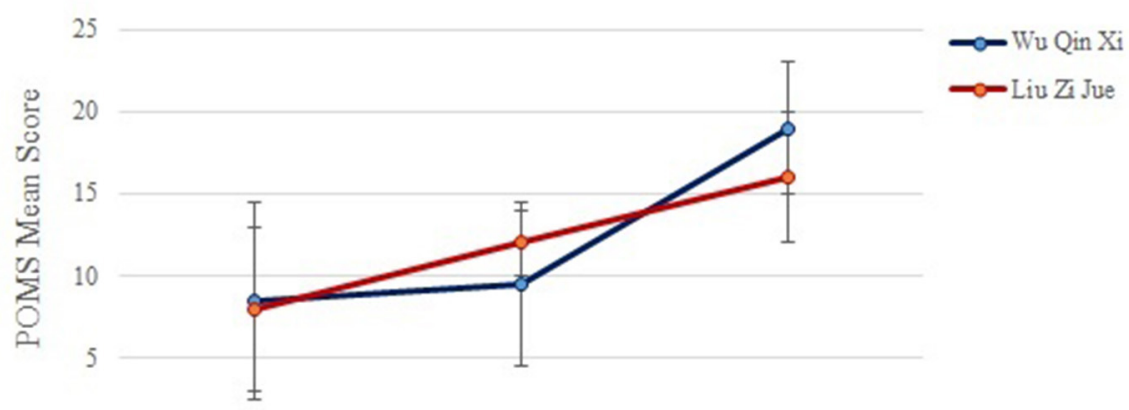

0
Pretest
15 Minutes
30 Minutes

B

\section{Fatigue-Inertia}

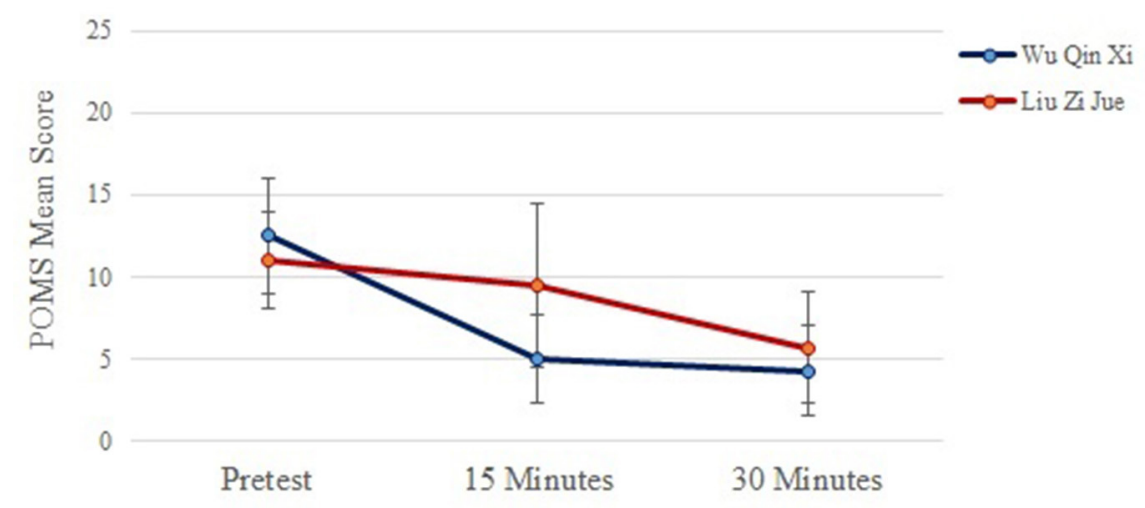

C

Tension-Anxiety

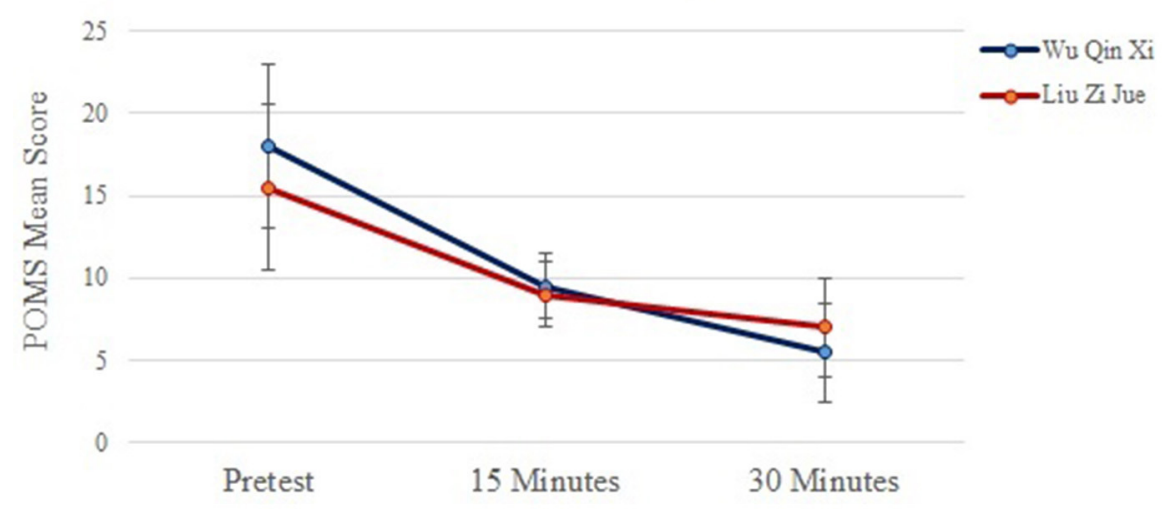

FIGURE 2 | (A-C) Means and SDs of the POMS scales vigor-activity, fatigue-inertia, and tension-anxiety in the dynamic Health Qigong techniques Wu Qin Xi and Liu Zi Jue at pretest, after $15 \mathrm{~min}$, and after $30 \mathrm{~min}$ of practice. Results show increased vigor-activity levels with decreased fatigue and tension-anxiety levels in both Health Qigong techniques after 30 min of practice. 
TABLE $2 \mid P$-values and partial eta-squared $\left(\eta_{p}{ }^{2}\right)$ for the data of the POMS-questionnaire for the factors Qigong technique, time, and the Qigong technique $\times$ time interaction for the scales vigor-activity, fatigue-inertia, and tension-anxiety.

\begin{tabular}{|c|c|c|c|c|c|c|}
\hline & \multicolumn{2}{|c|}{ Qigong technique } & \multicolumn{2}{|c|}{ Time } & \multicolumn{2}{|c|}{$\begin{array}{c}\text { Qigong technique } \mathrm{x} \\
\text { Time }\end{array}$} \\
\hline & $p$ & $\eta_{p}^{2}$ & $p$ & $\eta_{p}^{2}$ & $p$ & $\eta_{p}^{2}$ \\
\hline Vigor-activity & 0.022 & 0.18 & 0.034 & 0.10 & 0.042 & 0.07 \\
\hline Fatigue-inertia & n.s. & - & 0.028 & 0.14 & 0.040 & 0.08 \\
\hline Tension-anxiety & n.s. & - & 0.021 & 0.12 & n.s. & - \\
\hline
\end{tabular}

studies on static meditational Qigong can be observed. Dynamic Wu Qin Xi and Liu Zi Jue Qigong training induces a relaxed and attentive mind as indicated by an increase in midline frontocentral theta and posterior alpha-1, and alpha-2 activity after a practice duration of $30 \mathrm{~min}$. Thus, a training schedule with two practice cycles of $15 \mathrm{~min}$ of the movement sequences in $\mathrm{Wu}$ Qin $\mathrm{Xi}$ and $\mathrm{Liu} \mathrm{Zi}$ Jue is sufficient to approach the desired brain state. POMS data mirror the EEG findings with decreased fatigue and tension-anxiety scores in both techniques after $30 \mathrm{~min}$ of practice. We conclude that increases in EEG theta and alpha activity lead to a psychophysiological state of relaxation that reduces signs of fatigue and subjective sensations of tension and anxiety. Summarizing, a relaxing effect of the dynamic Qigong techniques $W u$ Qin $X i$ and $\mathrm{Liu} Z i$ Jue in sense of an evidence-based approach can be stated. Our results indicate that both dynamic Qigong techniques induce a centered and attentive state of mind indicated by increases in fronto-central midline theta activity. This psychophysiological brain state is clearly distinguishable from mind-wandering, i.e., thoughts are not remaining on the control of breathing and experience of bodily sensations but on task-unrelated contents. Empirical evidence shows that frontal EEG theta activity is activated in attentional processes and correlates negatively with the default mode network in resting state as it is activated in mind-wandering (Scheeringa et al., 2008). Summarizing, one main finding of the present study is that $W u$ Qin Xi and Liu Zi Jue induce comparable effects in EEG theta, alpha-1, and alpha-2 activity after $30 \mathrm{~min}$. Therefore, a relaxing effect and fostering of an attentive state of mind can be stated (Hong and Cho, 2012; Chou and Tsai, 2014).

The finding of increased fronto-central midline theta activity, and increased posterior alpha activity as shown in previous studies on the dynamic Qigong technique Wu Qin Xi (Henz et al., 2015; Henz and Schöllhorn, 2017) was replicated in the current study. Similar activations of brain activity are obtained in $\mathrm{Liu} \mathrm{Zi}$ Jue training. Our results are in line with previous studies on static meditational Qigong techniques. For instance, frontal mid-line theta rhythm during the concentrative Qigong state compared to the state of mind reached by non-concentrative Qigong meditation was shown by Pan et al. (1994). In the same manner, Shim (2012) demonstrated frontal theta activity after Qigong meditation in experienced practitioners. In a study conducted by Aftanas and Golocheikine (2001) EEG brain activity was examined in meditation in eyes-open and eyes-closed conditions. Depending on eyes-open and eyes-closed conditions, different patterns of anterior and midline theta activity occurred. The authors argue that the found theta activity reflects internalized attentional processes during meditation that are dependent on eyes-open and eyes-closed states. Considering EEG alpha activity, the findings are in line with previous studies that stated increases in EEG alpha activity in static meditational Qigong. According to previous studies (Zhang et al., 1988a,b; Yang et al., 1994; Jang et al., 2004; Qin et al., 2009; Faber et al., 2012), a shift of EEG activity from posterior to anterior regions was observed in $W u$ Qin Xi and Liu Zi Jue in the present study.

The highlighted finding of this study is that different temporal courses with different topographical patterns in spontaneous EEG brain activity in the frequency bands theta, alpha-1, alpha2, and gamma are obtained in Wu Qin Xi and Liu Zi Jue. Considering the temporal course of EEG activity in the alpha1 and alpha-2 band stronger fluctuations in $W u$ Qin $X i$ than in Liu Zi Jue are observed. In Liu Zi Jue continuous increases of alpha- 1 in frontal regions, and alpha- 2 activity in parietal regions is obtained after 15 and $30 \mathrm{~min}$, respectively. In $\mathrm{Wu}$ Qin Xi, a decrease compared to baseline rest of EEG alpha- 1 activity is obtained in central, and parietal regions after $15 \mathrm{~min}$, followed by an increase after $30 \mathrm{~min}$. A similar pattern of results is observed for alpha-2 activity. A significant decrease in alpha-2 activity is obtained after $15 \mathrm{~min}$, followed by an increase after $30 \mathrm{~min}$ in parietal areas. Considering theta activity, we obtained increases in parietal areas after $15 \mathrm{~min}$ in Liu Zi Jue compared to Wu Qin $X i$ training after $15 \mathrm{~min}$. In $W u$ Qin $X i$, occipital theta activity was increased after $30 \mathrm{~min}$, compared to $15 \mathrm{~min}$, and baseline rest. Gamma activity was observed only in Liu Zi Jue after $30 \mathrm{~min}$ in left central regions. POMS data on the vigor-activity scale show a significant technique by time interaction with a stronger fluctuation of vigor-activity levels in $W u$ Qin $X i$ during practice. This finding on the subjective psychophysiological state reflects the results obtained in the EEG alpha- 1 and alpha- 2 bands.

In summary, stronger fluctuations regarding the temporal course of alpha-1 and alpha-2 activity in Wu Qin Xi compared to Liu Zi Jue are demonstrated. In Liu Zi Jue, EEG alpha-1 and alpha-2 activity increased more quickly after 15 min than in $W u$ Qin Xi. Theta activity increased in parietal regions in $\mathrm{Liu} \mathrm{Zi}$ Jue. We discuss three lines of argumentation for the obtained results in the present study: (1) the role of enhanced breathing in Liu Zi Jue, (2) the role of cognitive and internalized attentional processes due to properties of the practice sequences, and (3) the interaction of attentional processes and motor control in both movement sequences.

From previous studies on Qigong meditation, we hypothesized that practicing the Qigong techniques $W u$ Qin $X i$ and Liu $\mathrm{Zi}$ Jue result in increases in frontal theta and posterior alpha activity. According to the theoretical framework of TCM, we hypothesized that practicing the Qigong technique Liu Zi Jue leads to stronger increases in EEG theta activity as a correlate for a concentrative meditational state compared to practice of the Qigong technique $W u$ Qin Xi. From a physiological perspective, we argue that in Liu $\mathrm{Zi}$ Jue the role of breathing is more reinforced due to producing the six sounds when performing the movements. Therefore, breathing behavior tends to be more regulated by adapting to the sound production than in $W u$ Qin 
$X i$. Recent EEG studies have shown that abdominal breathing techniques lead to increased frontal theta activity (e.g., Yu et al., 2011; Chervin et al., 2012; Park and Park, 2012). Considering breathing as a meditation technique, it was observed that Shaolin Dan Tian Breathing increases EEG frontal theta activity (Chan et al., 2011). The authors argue that the observed increase in frontal theta activity in Shaolin Dan Tian Breathing is a correlate for an attentive mind. Further, several studies have shown that abdominal breathing enhances EEG alpha activity. For instance, Arambula et al. (2001) demonstrated increases in EEG alpha activity in abdominal breathing techniques. Increased alpha band activity with decreased theta band activity was obtained in abdominal breathing during Zen practice (Arita, 2012). Comparing alpha-1 and alpha-2 activity Fumoto et al. (2004) showed increases in alpha-1 activity with disappearance of alpha-2 activity in voluntary abdominal breathing. From these results we argue that the found pattern of activation in the alpha-1 and alpha-2 band with stronger increases after $15 \mathrm{~min}$ in Liu Zi Jue compared to $W u$ Qin Xi, reflect modulations of EEG brain activity due to the stronger reinforced attention on breathing in Liu Zi Jue than in Wu Qin Xi. The pattern of decreased EEG alpha-1 and alpha-2 activity after $15 \mathrm{~min}$, and delayed increase after $30 \mathrm{~min}$ in $\mathrm{Wu}$ Qin Xi possibly mirrors differences in breathing behavior due to the practice structure with less control of breathing capacities by sound production as it is prescribed in Liu Zi Jue.

A second line of argumentation for a stronger increase in EEG theta activity in $\mathrm{Liu} \mathrm{Zi}$ Jue than in $\mathrm{Wu} \mathrm{Qin} \mathrm{Xi}$ is that attentional processes are more guided by directing the focus of attention on breathing due to sound production than on mere movement performance. This might lead to stronger internalized attentional processing that mirrors in increased EEG frontal theta activity in Liu Zi Jue. In contrast, Wu Qin Xi affords less binding of breathing capacities. Further, movement sequences afford high capacities in spatial processing. This Qigong technique requires practitioners to move spatially. Spatial directions and distances are strongly determined which in consequence force practitioners to direct their attention at least partially to the external space to perform the movement sequences appropriately. We argue that the focus of attention during practicing the movement sequences mediates the effects on EEG brain activity. For instance, Van der Lubbe et al. (2014) showed that the focus of spatial attention leads to changes in EEG brain activity. From behavioral studies on the role of attentional performance it is known that an external focus of attention alleviates movement performance, and therefore requires less effort. For instance, it was shown that movement performance benefits from an external focus of attention in gymnastics (Abdollahipour et al., 2015). Several recent studies have provided evidence that movement efficiency is enhanced by an external focus (Zachry et al., 2005; Marchant et al., 2009). Benefits of directing attention to an external focus have been found to result in more effective motor performance than those inducing an internal focus by directing attention to the body movements themselves (Totsika and Wulf, 2003; Wulf et al., 2003, 2009; Wulf, 2007). It is argued that focusing on the intended movement effect facilitates the utilization of unconscious or automatic processes. This results in greater movement ease or fluidity (Wulf et al., 2001; Wulf and Lewthwaite, 2010). In contrast, focusing on one's own movements leads to a more conscious type of movement control, thereby constraining the motor system and disrupting automatic control processes (Wulf et al., 2001). It has been shown that relative to an internal focus, an external focus reduces attentional demands and results in the utilization of fast reflexive feedback loops (Wulf et al., 2001). Transferring these findings on the dynamic Qigong techniques examined in the current study, might explain the found differences in the temporal course of EEG theta and alpha1 and alpha-2 activity in $W u$ Qin Xi and Liu Zi Jue, Liu Zi Jue induces a strongly internally directed attentional processing due to binding of breathing capacities on sound production. In contrast, Wu Qin Xi affords a strongly spatial processing due to moving in space to spatial directions, and due to its complex movement configurations.

From this line of argumentation, an important question arises that opens our third line of argumentation: does an internal focus of attention during dynamic Health Qigong practice lead to a more demanding type of movement control, and therefore binds more attention which finally results in increased EEG frontal theta activity? Especially in novice practitioners, an attentionally demanding motor learning process during Health Qigong practice could result in enhanced stress reduction mirrored by changes in EEG brain activity. We argue that one underlying cognitive mechanism is a working memory load which results from increased motor affordances during dynamic Health Qigong practice. From a neurophysiological point of view, frontal theta power has been found to increase with working memory load (Gevins et al., 1997; Jensen and Tesche, 2002; Onton et al., 2005). Challenging working memory may lead to a loss of a merely executive action control due to limited resource capacity. As a consequence, practitioners' attention is drawn away from cognitive engagement in everyday thoughts by a demanding movement control process in dynamic Health Qigong training (see Schmalzl et al., 2014). Further, a loss of cognitive action control toward a state of non-focusing and non-involvement on the everyday mind flow is one of the main aims in Eastern meditation techniques (for an overview see Tomasino et al., 2014). Especially in Buddhism-related meditation traditions a mindfulness state is reached by sustained attention on the body. Activations in midline fronto-central lobe structures associated with attentional processes possibly confirming the fundamental role of mindfulness shared by many Buddhist meditations (for an overview see Tomasino et al., 2014). Transferring these findings on the results obtained in the current study, we infer that different cognitive and attentional processes lead to the obtained temporal courses in EEG brain activity in $W u$ Qin $X i$ and Liu Zi Jue. While the state of internalized attention in Liu $\mathrm{Zi}$ Jue is reached by directing the focus of attentional processing internally due to binding of breathing capacities in Liu Zi Jue, a psychophysiological state of relaxation is reached delayed in $W u$ Qin $X i$ after 30 min. Possibly, executive control processes of the complex movement configurations rather than by breathing behavior mediated internalized attention play the main part mirrored in reduced alpha- 1 and alpha- 2 activity after $15 \mathrm{~min}$. Thus, directing attention externally toward the 
movement, might lead to a more superficial movement execution without focusing breathing and on bodily sensations, which is mirrored in decreases in theta and alpha power.

Finally, movement configurations in Liu Zi Jue are reported from practitioners to be less complex than in $W u$ Qin $X i$. Therefore, practitioners possibly have more attentional capacities to spend effort on control of breathing in Liu Zi Jue than on control of complex movement configurations as in Wu Qin Xi. Following this line of argumentation, the obtained results of increased theta, alpha-1 and alpha- 2 activity can be interpreted in this manner.

To date, there are no systematical studies reported on the effects of Liu Zi Jue Qigong training on EEG brain activity. Recently, it was shown that Liu Zi Jue training made beneficial effects on pain consciousness and depression of elderly single women (Dong and Lee, 2013). As depression is correlated with reduced EEG alpha activity (see Başar et al., 2012), the present study gives insight into the underlying neurophysiological processes that mediate the beneficial effects of Liu Zi Jue in depression. Liu Zi Jue training increases low-frequency EEG (theta and alpha-1 activity) that regulate the brain activity of reduced alpha activity in depression patients down to the level of healthy subjects.

To our knowledge, the present study is the first one that compares general acute effects and the temporal course of dynamic Qigong Wu Qin Xi and Liu Zi Jue training on EEG brain activity. Summarizing, a relaxation effect in $W u$ Qin $X i$ and Liu Zi Jue in a sense of an evidence-based approach is to be stated: the dynamic Qigong techniques $W u$ Qin $X i$ and $\mathrm{Liu} \mathrm{Zi}$ Jue induce increased midline fronto-central theta and shifts of alpha activity from posterior to anterior regions after $30 \mathrm{~min}$ of training. Thus, we obtained comparable patterns and intensities of EEG brain activity after $30 \mathrm{~min}$ in both Qigong techniques. Different temporal courses in EEG theta, alpha-1, and alpha-2 activity were demonstrated. In Liu Zi Jue, a continuous increase in EEG fronto-central theta, posterior alpha-1, and alpha-2 activity was obtained, whereas in $W u$ Qin $X i$ decreases of central and parietal alpha-1, and central alpha- 2 activity was demonstrated after $15 \mathrm{~min}$, followed by an increase after $30 \mathrm{~min}$.

\section{REFERENCES}

Abbott, R., and Lavretsky, H. (2013). Tai chi and Qigong for the treatment and prevention of mental disorders. Psychiatr. Clin. N. Am. 36, 109-110. doi: 10.1016/j.psc.2013.01.011

Abdollahipour, R., Wulf, G., Psotta, R., and Palomo Nieto, M. (2015). Performance of gymnastics skill benefits from an external focus of attention. J. Sports Sci. 33, 1807-1813. doi: 10.1080/02640414.2015.1012102

Aeschbach, D., Matthews, J. R., Postolache, T. T., Jackson, M. A., Giesen, H. A., and Wegr, T. A. (1999). Two circadian rhythms in the human electroencephalogram during wakefulness. Am. J. Physiol. 277, R1771-R1779.

Aftanas, L. I., and Golocheikine, S. A. (2001). Human anterior and frontal midline theta and lower alpha reflect emotionally positive state and internalized attention: high-resolution EEG investigation of meditation. Neurosci. Lett. 310, 57-60. doi: 10.1016/S0304-3940(01)02094-8

Arambula, P., Peper, E., Kawakami, M., and Gibney, K. H. (2001). The physiological correlates of Kundalini Yoga meditation: a study of a yoga
We argue that the differences regarding the temporal course of EEG brain activity result from a tighter control of breathing behavior during sound production in Liu Zi Jue. Further, we hypothesize that the control of performance of complex movement sequences in $W u$ Qin Xi leads to the observed pattern of EEG brain activity.

The results of our study have important implications for the design of interventions applying the dynamic Qigong techniques Wu Qin Xi and Liu Zi Jue especially with the indication for a strengthening of mental health and stress reduction. Especially in clinical populations who display reduced spontaneous alpha activity as in stress mediated diseases like burnout (van Luitjelaar et al., 2010; Tukaiev et al., 2012), but as well as in anxiety, depression, and bipolar disorders (Başar et al., 2012) a strong induction of alpha activity by Qigong practice is essential for the therapeutic success of the intervention. The data on the subjective psychophysiological state underline the reducing effect of both dynamic Qigong techniques on signs of tension, anxiety, and fatigue. Therefore, it is recommended, to practice $W u$ Qin $X i$ for $30 \mathrm{~min}$ to reach a psychophysiological effect of relaxation and attentiveness. In Liu Zi Jue a practice session of at least $15 \mathrm{~min}$ is recommended. Further research is needed to clarify the role of long-term training effects in both Qigong techniques on the temporal course of EEG brain activity. One further interesting question is whether the same effects and temporal courses in brain activity would be expected in clinical populations that show reduced alpha oscillations at resting baseline.

\section{AUTHOR CONTRIBUTIONS}

The authors DH and WS cooperated on developing the theoretical framework and preparing the manuscript.

\section{ACKNOWLEDGMENTS}

This work was supported by the Inneruniversitäre Forschungsförderung of the Johannes Gutenberg University of Mainz (Germany). master. Appl. Psychophysiol. Biofeedback 26, 147-153. doi: 10.1023/A:10113433 07783

Arita, H. (2012). Anterior prefrontal Cortes and serotonergic system activation during meditation practice induces negative mood improvement and increased alpha band in EEG. Rinsho Shinkeigaku 52, 1279-1280.

Başar, E., Güntekin, B., Atagün, I., Turp Gölbasi, B., Tülay, E., and Özerdem, A. (2012). Brain's alpha activity is highly reduced in euthymic bipolar disorder patients. Cogn. Neurodyn. 6, 11-20. doi: 10.1007/s11571-011-9172-y

Biesinger, E., Kipman, U., Schatz, S., and Langguth, B. (2010). Qigong for the treatment of tinnitus: a prospective randomized controlled study. J. Psychosom. Res. 69, 299-304. doi: 10.1016/j.jpsychores.2010.04.013

Cantero, J. L., Atienza, M., and Salas, R. M. (2002). Human alpha oscillations in wakefulness, drowsiness period, and REM sleep: different electroencephalographic phenomena within the alpha band. Neurophysiol. Clin. 32, 54-71. doi: 10.1016/S0987-7053(01)00289-1

Chan, A. S., Cheung, M. C., Sze, S. L., Leung, W. W., and Shi, D. (2011). Shaolin dan tian breathing fosters relaxed and attentive mind: a randomized 
controlled neuro-electrophysiological study. Evid. Based Complement. Altern. Med. 2011:180704. doi: 10.1155/2011/180704

Chan, A. S., Cheung, M. C., Chan, Y. L., Yeungt, D. K., and Lam, W. (2006). Bilateral frontal activation associated with cutaneous stimulation of Elixir Field: an fMRI study. Am. J. Chin. Med. 34, 207-216. doi: $10.1142 / \mathrm{S} 0192415 \mathrm{X} 06003771$

Chan, J. S., Ho, R. T., Wang, C. W., Yuen, L. P., Sham, J. S., and Chan, C. L. (2013). Effects of qigong exercise on fatigue, anxiety, and depressive symptoms of patients with chronic fatigue syndrome-like illness: a randomized controlled trial. Evid. Based Complement. Altern. Med. 2013:485341. doi: 10.1155/2013/485341

Chen, Y. (2011). Effect of "WuQinXi" exercise on blood lipid levels and the antioxidant enzyme activities in aged practicers. Afr. J. Microbiol. Res. 5, 5733-5736.

Cheng, R. W., Borrett, D. S., Cheng, W., Kwan, H. C., and Cheng, R. S. (2010). Human prefrontal cortical response to the meditative state: a spectroscopy study. Int. J. Neurosci. 120, 483-488. doi: 10.3109/00207454.2010. 483650

Chervin, R. D., Shelgikar, A. V., and Burns, J. W. (2012). Respiratory cycle-related EEG changes: response to CPAP. Sleep 35, 203-209. doi: 10.5665/sleep.1622

Cheung, B. M., Lo, J. L., Fong, D. Y., Chan, M. Y., Wong, S. H., Wong, V. C., et al, (2005). Randomised controlled trial of qigong in the treatment of mild essential hypertension. J. Hum. Hypertens. 19, 697-704. doi: 10.1038/si.jhh.1001884

Chinese Health Qigong Association (2007). Chinese Health Qigong - Liu Zi Jue. Beijing: Chinese Health Qigong Association.

Chou, W. L., and Tsai, M. H. (2014). Qigong dandao meditation improves visual attention. Iperception 5, 395-395.

Dong, L., and Lee, J.-B. (2013). The effects of health qigong training of elderly single women on pain consciousness and depression. Int. J. Appl. Sports Sci. 25, 118-126. doi: 10.24985/ijass.2013.25.2.118

Faber, P. L., Lehmann, D., Tei, S., Tsujiuchi, T., Kumano, H., Pascual-Marqui, R. D., et al. (2012). EEG source imaging during two Qigong meditations. Cogn. Process. 13, 255-265. doi: 10.1007/s10339-012-0441-4

Fumoto, M., Sato-Suzuki, I., Sekim, Y., Mohri, Y., and Arita, H. (2004). Appearance of high-frequency alpha band with disappearance of lowfrequency alpha band in EEG is produced during voluntary abdominal breathing in an eyes-closed condition. Neurosci. Res. 50, 307-317. doi: 10.1016/j.neures.2004.08.005

Gevins, A., Smith, M. E., McEvoy, L., and Yu, D. (1997). High-resolution EEG mapping of cortical activation related to working memory: effects of task difficulty, type of processing, and practice. Cereb. Cortex 7, 374-385. doi: 10.1093/cercor/7.4.374

Glei, D. A., Goldman, N., Lin, Y. H., and Weinstein, M. (2012). Relaxation practice and physiologic regulation in a national sample of older Taiwanese. J. Altern. Complement. Med. 18, 653-661. doi: 10.1089/acm.2010.0598

Grodin, M. A., Piwowarczyk, L., Fulker, D., Bazazi, A. R., and Saper, R. B. (2008). Treating survivors of torture and refugee trauma: a preliminary case series using Qigong and T'ai Chi. J. Altern. Complement. Med. 14, 801-806. doi: $10.1089 / \mathrm{acm} .2007 .0736$

Henz, D., and Schöllhorn, W. I. (2017). EEG brain activity in dynamic Health Qigong training: same effects for mental practice and physical training? Front. Psychol. 8:154. doi: 10.3389/fpsyg.2017.00154

Henz, D., Harbauer, M., and Schöllhorn, W. I. (2015). EEG alpha activity in novice and advanced Qigong practitioners before and after a six-week Qigong intervention. Psychophysiology 52:S68.

Henz, D., Tänny, P., and Schöllhorn, W. I. (2014). Attentional effects of practicing Qigong with and without music: an EEG study. Psychophysiology 51:S35.

Hong, S. S., and Cho, S. H. (2012). Effects of mindfulness-based qigong for children's concentration ability. Korean Soc. Orient. Neuropsychiatry 23, 49-58. doi: 10.7231/JON.2012.23.2.049

Hwang, E. Y., Chung, S. Y., Cho, J. H., Song, M. Y., Kim, S., and Kim, J. W. (2013). Effects of a brief qigong-based stress reduction program (BQSRP) in a distressed Korean population: a randomized trial. BMC Complement. Altern. Med. 13:113. doi: 10.1186/1472-6882-13-113

Jang, K., Choi, C., and Su, N. C. (2004). The studies on Qigong state using EEG, fMRI, EAV and SQUID measurements. Korean J. Acupunct. 21, 1-28.

Jensen, O., and Tesche, C. D. (2002). Frontal theta activity in humans increases with memory load in a working memory task. Eur. J. Neurosci.15, 1395-1399. doi: 10.1046/j.1460-9568.2002.01975.x
Jiang, Y., and Zou, J. (2013). Analysis of the TCM theory of traditional Chinese health exercise. J. Sport Health Sci. 2, 204-208. doi: 10.1016/j.jshs.2013.03.008

Kim, S. H., Schneider, S. M., Kravitz, L., Mermier, C., and Burge, M. R. (2013). Mind-body practices for posttraumatic stress disorder. J. Investig. Med. 61, 827-834. doi: 10.2310/JIM.0b013e3182906862

Lavallee, C. F. Hunter, M. D., and Persinger, M. A. (2011). Intracerebral source generators characterizing concentrative meditation. Cogn. Process. 12, 141-150. doi: 10.1007/s10339-011-0394-z

Lee, M. S., Bae, B. H., Ryu, H., Sohn, J. H., Kim, S. Y., and Chung, H. T. (1997). Changes in alpha wave and state anxiety during ChunDoSunBup Qi-training in trainees with open eyes. Am. J. Chin. Med. 25, 289-299. doi: 10.1142/S0192415X97000329

Lee, M. S., Kang, C. W., Lim, H. J., and Lee, M. S. (2004). Effects of Qi-training on anxiety and plasma concentrations of cortisol, ACTH, and aldosterone: a randomized placebo-controlled pilot study. Stress Health 20, 243-248. doi: $10.1002 /$ smi. 1023

Lee, M. S., Kim, B. G., Huh, H. J., Ryu, H., Lee, H. S., and Chung, H. T. (2000). Effect of Qi-training on blood pressure, heart rate and respiration rate. Clin. Rehabil. 23, 504-511. doi: 10.1046/j.1365-2281.2000.00242.x

Lee, M. S., Lee, M. S., Kim, H. J., and Moon, S. R. (2003). Qigong reduced blood pressure and catecholamine levels of patients with essential hypertension. Int. J. Neurosci. 113, 1691-1701. doi: 10.1080/00207450390245306

Lehmann, D., Faber, P. L., Tei, S., Pascual-Marqui, R. D., Milz, P., and Kochi, K. (2012). Reduced functional connectivity between cortical sources in five meditation traditions detected with lagged coherence using EEG tomography. Neuroimage 60, 1574-1586. doi: 10.1016/j.neuroimage.2012.01.042

Liu, X., Clark, J., Siskind, D., Williams, G. M., Byrne, G., Yang, J. L., et al. (2015). A systematic review and meta-analysis of the effects of Qigong and Tai Chi for depressive symptoms. Complement. Ther. Med. 23, 516-534. doi: $10.1016 /$ j.ctim.2015.05.001

Marchant, D. C., Greig, M., and Scott, C. (2009). Attentional focusing instructions influence force production and muscular activity during isokinetic elbow flexions. J. Strength Cond. Res. 23, 2358-2366. doi: 10.1519/JSC.0b013e3181b8d1e5

Martínez, N., Martorell, C., Espinosa, L., Marasigan, V., Domenech, S., and Inzitari, M. (2015). Impact of Qigong on quality of life, pain and depressive symptoms in older adults admitted to an intermediate care rehabilitation unit: a randomized controlled trial. Aging Clin. Exp. Res. 27, 125-130. doi: 10.1007/s40520-014-0250-y

McNair, D. M., Lorr, M., and Droppleman, L. F. (1971). Edits Manual. Profile of Mood States. San Diego, CA: Educational and Industrial Testing Service.

Ng, B. H., and Tsang, H. W. (2009). Psychophysiological outcomes of health qigong for chronic conditions: a systematic review. Psychophysiology 46, 257-269. doi: 10.1111/j.1469-8986.2008.00763.x

Onton, J., Delorme, A., and Makeig, S. (2005). Frontal midline EEG dynamics during working memory. Neuroimage 27, 341-356. doi: 10.1016/j.neuroimage.2005.04.014

Pan, W., Zhang, L., and Xia, Y. (1994).The difference in EEG theta waves between concentrative and non-concentrative qigong states-a power spectrum and topographic mapping study. J. Tradit. Chin. Med. 14, 212-218.

Park, Y. J., and Park, Y. B. (2012). Clinical utility of paced breathing as a concentration meditation practice. Complement. Ther. Med. 20, 393-399. doi: 10.1016/j.ctim.2012.07.008

Posadzki, P., Parekh, S., and Glass, N. (2010). Yoga and Qigong in the psychological prevention of mental health disorders: a conceptual synthesis. Chin. J. Integr. Med. 16, 80-86. doi: 10.1007/s11655-009-9002-2

Qin, Z., Yin, J., Lin, S., and Hermanowicz, N. S. (2009). A forty-five year follow-up EEG study of qigong practice. Int. J. Neurosci. 119, 538-552. doi: $10.1080 / 00207450802325520$

Scheeringa, R., Bastiaansen, M. C., Petersson, K. M., Oostenveld, R., Norris, D. G., and Hagoort, P. (2008). Frontal theta EEG activity correlates negatively with the default mode network in resting state. Int. J. Psychophysiol. 67, 242-251. doi: 10.1016/j.ijpsycho.2007.05.017

Schmalzl, L., Crane-Godreau, M. A., and Payne, P. (2014). Movement-based embodied contemplative practices: definitions and paradigms. Front. Hum. Neurosci. 8:205. doi: 10.3389/fnhum.2014.00205

Shim, J. (2014). Effects of Qigong exercise program on smoking cessation selfefficacy, stress and EEG concentration indices of adolescent smokers. Youth Facilit. Environ. 12, 163-174. 
Shim, J. (2012). Comparison of EEG characteristics between short and long-term practitioners during Qigong practice. J. Korean Allian. Mart. Arts 14, 209-228.

Sousa, M. C., Goncalves, M., Machado, J., Efferth, T., Greten, T., Froeschen, P., et al. (2012). Effects of qigong on performance-related anxiety and physiological stress functions in transverse flute music schoolchildren: a feasibility study. $J$. Chin. Integr. Med. 10, 858-865. doi: 10.3736/jcim20120805

Stenlund, T., Ahlgren, C., Lindahl, B., Burell, G., Steinholtz, K., Edlund, C., et al. (2009). Cognitively oriented behavioral rehabilitation in combination with Qigong for patients on long-term sick leave because of burnout: REST - a randomized clinical trial. Int. J. Behav. Med. 16, 294-303. doi: 10.1007/s12529-008-9011-7

Stenlund, T., Nordin, M., and Järvholm, L. S. (2012). Effects of Rehabilitation programmes for patients on long-term sick leave for burnout: a 3-year followup of the rest study. J. Rehabil. Med. 44, 684-690. doi: 10.2340/16501977-1003

Sun, Y. (1988). The influence of Qigong and Taijiquan in respiratory rehabilitation. Chin. J. Rehabil. 4:168.

Tei, S., Faber, P., and Lehmann, D. (2006a). Experience influences the EEG-theta localizations in QiGong meditation. Int. J. Psychophysiol. 61, 367-368.

Tei, S., Faber, P., Lehmann, D., Tsujiuchi, T., Pascual-Marqui, R. D., and Kumano, H. (2009). EEG frequency-band LORETA distinguishes meditation from resting in Qigong meditators. Neuropsychobiology 59, 65-66.

Tei, S., Faber, P. L., Lehmann, D., Shibata, I., Ohyama, G., Tsujiuchi, T., et al. (2006b). Magnitude and location of EEG spectral power in experienced QiGong meditators during resting and three meditations. J. Psychosom. Res. 61, $420-420$.

Terjestam, Y., Jouper, J., and Johansson, C. (2010). Effects of scheduled Qigong exercise on pupils' well-being, self-image, distress, and stress. J. Altern. Complement. Med. 16, 939-944. doi: 10.1089/acm.2009.0405

Tomasino, B., Chiesa, A., and Fabbro, F. (2014). Disentangling the neural mechanisms involved in Hinduism- and Buddhism-related meditation. Brain Cogn. 90, 32-40. doi: 10.1016/j.bandc.2014.03.013

Totsika, V., and Wulf, G. (2003). The influence of external and internal foci of attention on transfer to novel situations and skills. Res. Q. Exerc. Sport 74, 220-225. doi: 10.1080/02701367.2003.10609084

Tsang, H. W., Cheung, I., and Lak, D. C. (2002). Qigong as a psychosocial intervention for depressed elderly with chronic physical illness. Int. J. Geriatr. Psychiatry 17, 1146-1154. doi: 10.1002/gps.739

Tsang, H. W., Mok, C. K., Au Yeung, Y. T., and Chan, S. Y. (2003). The effect of Qigong on general and psychological health of elderly with chronic physical illnesses. A randomized clinical trial. Int. J. Geriatr. Psychiatry 18, 441-449. doi: $10.1002 /$ gps.861

Tukaiev, S., Krizhanovskij, S., Zima, I., Filimonova, N., Radchuk, O., Cherninskiy, A., et al. (2012). Gender-related differences in spatial synchronization in EEG in burnout students. Psychophysiology 49:120.

Van der Lubbe, R. H., Bundt, C., and Abrahamse, E. L. (2014). Internal and external spatial attention examined with lateralized EEG power spectra. Brain Res. 1583, 179-192. doi: 10.1016/j.brainres.2014.08.007

van Luitjelaar, G., Verbraak, M., van den Bunt, M., Keijsers, G., and Arns, M. (2010). EEG findings in burnout patients. J. Neuropsychiatry Clin. Neurosci. 22, 208-217. doi: 10.1176/jnp.2010.22.2.208

Wallace, R. K. (1970). Physiological effects of transcendental meditation. Science 167, 1751-1754. doi: 10.1126/science.167.3926.1751

Wang, C. W., Chan, C. L., Ho, R. T., Tsang, H. W., Chan, C. H., and Ng, S. M. (2013). The effect of qigong on depressive and anxiety symptoms: a systematic review and meta-analysis of randomized controlled trials. Evid. Based Complement. Altern. Med. 2013:716094. doi: 10.1155/2013/716094

Wang, C. W., Chan, C. H., Ho, R. T., Chan, J. S., Ng, S. M., and Chan, C. L. (2014). Managing stress and anxiety through qigong exercise in healthy adults: a systematic review and meta-analysis of randomized controlled trials. Complement. Altern. Med. 14:8. doi: 10.1186/1472-6882-14-8

Wang, F., Man, J. K. M., Lee, E.-K. O., Wu, T., Benson, H., and Fricchione, G. L. (2013). The effects of qigong on anxiety, depression, and psychological wellbeing: a systematic review and meta-analysis. Evid. Based Complement. Altern. Med. 2013:152738. doi: 10.1155/2013/152738

Wulf, G. (2007). Attention and Motor Skill Learning. Champaign, IL: Human Kinetics.

Wulf, G., Landers, M., Lewthwaite, R., and Töllner, T. (2009). External focus instructions reduce postural instability in individuals with Parkinson disease. Phys. Ther. 89, 162-168. doi: 10.2522/ptj.20080045
Wulf, G., McNevin, N. H., and Shea, C. H. (2001). The automaticity of complex motor skill learning as a function of attentional focus. Q. J. Exp. Psychol. A 54, 1143-1154. doi: 10.1080/713756012

Wulf, G., Weigelt, M., Poulter, D., and McNevin, N. (2003). Attentional focus on suprapostural tasks affects balance learning. Q. J. Exp. Psychol. A 56, 1191-1211. doi: $10.1080 / 02724980343000062$

Wulf, G., and Lewthwaite, R. (2010). "Effortless motor learning? An external focus of attention enhances movement effectiveness and efficiency," in Effortless Attention: A New Perspective in Attention and Action, ed B. Bruya (Cambridge, MA: MIT Press), 75-101.

Xiao, C. M., and Zhuang, Y. C. (2015). Efficacy of liuzijue qigong in individuals with chronic obstructive pulmonary disease in remission. J. Am. Geriatr. Soc. 63, 1420-1425. doi: 10.1111/jgs.13478

Yang, S. H., Yang, Q. F., and Shi, J. M. (1994). Observation of electroencephalogram spectrum changes over one year of Qigong training. Zhongguo Zhong Xi Yi Jie He Za Zhi 14, 643-646.

Yang, Y.-W., and Wu, H.-Z., (2011). Origin and development of [qigong-wuqinxi]. Zhonghuayishizazhi 41, 265-267.

Yeom, S., Kwon, Y., and Woo, J. H. (2013). Study on the Movement of New Qigong "WuQinXi" Exercise for Lumbar Spinal Disease: Based on 10 Mode, 15 Mode, 18 Mode. Korean J Orient. Physiol. Pathol. 27, 280-288.

Yeung, A., Slipp, L. E., Jacquart, J., Fava, M., Denninger, J. W., Benson, H., et al. (2013). The treatment of depressed Chinese Americans using Qigong in a health care setting: a pilot study. Evid. Based Complement. Altern. Med. 2013:168784. doi: 10.1155/2013/168784

Yin, J., and Dishman, R. K. (2014). The effect of Tai Chi and Qigong practice on depression and anxiety symptoms: a systematic review and meta-regression analysis of randomized controlled trials. Ment. Health Phys. Act. 7, 135-146. doi: 10.1016/j.mhpa.2014.08.001

You, K. G., Kwon, Y. D., and Jeong, H. W. (2014). Study on the movement of new Qi-gong "WuQinXi" exercise for lumbar spinal disease: based on 20 mode, 30 mode, 40 mode. Korean J. Orient. Physiol. Pathol. 28, 129-136. doi: 10.15188/kjopp.2014.04.28.2.129

You, K. G., Yeom, S. R., Lee, S. Y., Kwon, Y. D., and Jeong, H. W. (2013). Study on the movement of new Qi-gong "WuQinXi" exercise for lumbar spinal disease: based on 10 mode, 15 mode, 18 mode. Korean J. Orient. Physiol. Pathol. 27, $280-288$.

Yu, W. L., Li, X. Q., Tang, W. J., Li, Y., Weng, X. C., and Chen, Y. Z. (2007). fMRI study of pain reaction in the brain under state of "Qigong." Am. J. Chin. Med. 35, 937-945. doi: 10.1142/S0192415X07005405

Yu, X., Fumoto, M., Nakatani, Y., Sekiyama, T., Kikuchi, H., Seki, Y., et al. (2011). Activation of the anterior prefrontal cortex and serotonergic system is associated with improvements in mood and EEG changes induced by Zen meditation practice in novices. Int. J. Psychophysiol. 80, 103-111. doi: 10.1016/j.ijpsycho.2011.02.004

Zachry, T., Wulf, G., Mercer, J., and Bezodis, N. (2005). Increased movement accuracy and reduced EMG activity as the result of adopting an external focus of attention. Brain Res. Bull. 67, 304-309. doi: 10.1016/j.brainresbull.2005.06.035

Zhang, F., Bai, Y. H., and Zhang, J. (2014). The Influence of "WuQinXi" exercises on the Lumbosacral Multifidus. J. Phys. Ther. Sci. 26, 881-884. doi: $10.1589 /$ jpts.26.881

Zhang, J. Z., Li, J. Z., and He, Q. N. (1988a). Statistical brain topographic mapping analysis for EEGs recorded during Qi Gong state. Int. J. Neurosci. 38, $415-425$.

Zhang, J. Z., Zhao, J., and He, Q. N. (1988b). EEG Findings during special psychical state (Qigong state) by means of compressed spectral array and topographic mapping. Comput. Biol. Med. 18, 455-463. doi: 10.1016/0010-4825(88)90 063-7

Conflict of Interest Statement: The authors declare that the research was conducted in the absence of any commercial or financial relationships that could be construed as a potential conflict of interest.

Copyright $\odot 2018$ Henz and Schölhorn. This is an open-access article distributed under the terms of the Creative Commons Attribution License (CC BY). The use, distribution or reproduction in other forums is permitted, provided the original author(s) or licensor are credited and that the original publication in this journal is cited, in accordance with accepted academic practice. No use, distribution or reproduction is permitted which does not comply with these terms. 